\title{
Characterization of Doggy Style Sex-Alcohol Dependent Inter Subtype Among Men Who Have Sex with Women Lead in Heart Disease for Men From India
}

\author{
Rahul Hajare* \\ Indian council of Medical Research, National AIDS Research Institute, Delhi, India
}

Received: 琒July 18, 2018; Published: 阱July 25, 2018

*Corresponding author: Rahul Hajare, Post Doc Scholar, Indian council of Medical Research, National AIDS Research Institute, Delhi, India

\begin{abstract}
We report here doggy style sex inter subtype alcohol dependent recombinants from India. One among those is recombinant penile fracture is reported for the first time from India.

\section{Statement}

Wrinkle shows life is loved your life and ageing with dignity. Indian men took a lot of hydroxyl drugs trying to keep her weigh down and look younger. These drugs reduce the hunger and heighten the metabolic rate and makes heart beat faster while having sex in consist doggy style. Men who have experienced intimate health disease are at greater risk for physical and mental health problems including posttraumatic stress disorder (PTSD) and alcohol dependency. Most men get a severe stroke and the impact of all may compound these costs. Researchers have reported that men with these experiences are more difficult to treat; many do not access treatment and those who do, frequently do not stay because of difficulty maintaining helping relationships and hesitation. However, these men's perspective has not been previously studied in close monitoring. The purpose of this study is to describe the experience of seeking help for hydroxyl drugs dependency by Indian men for sex and food for bedtime to avoid the present age. Men with hydroxyl drugs dependency with a history of depression want help however the health and social services do not always recognize their calls for help or their symptoms of distress. Drug dependency hydroxyl drugs thickens and stiffens artery walls, which can inhibit blood flow through arteries to organs and tissues that involved narrowed or blocked blood vessels that can lead to a heart attack and over sex.
\end{abstract}

\section{Introduction}

Unsymmetrical human has incompatible essential genes and hormone one of the highest and unrivaled of the divine attributes, or powers and energies. Human structure has built upon on nonpatented small molecule, it has it could be because of genes and hormones cannot ignored. That can why dark circles can be so hard to reduce. Dark circles do not just form because of lack of sleep. It could be because of your genes and hormones exchange while having sex $[1,2]$. That is why dark circles can be so hard to reduce. New study has recommended about $41 \%$ of penile fracture in men post adolescent. The research titled, "relationship between sexual position and severity of penile fracture and safe sex: the train your mind (revise)" published earlier placed the ancient missionary position as the second most dangerous sex position causing about $25 \%$ of penile fracture cases. Researchers, from London The Hindu University who did the study found doggy style and missionary

position could cause equally serious penile fracture. Extended recommendation that the sex position in which the woman sits on top of the man is the safest. It is a meaty matter! Why the debate on eating dog meat in India (and globally) is hypocritical lead doggy style position $[3,4]$. "We do not observe differences between the severity of the penile fracture between the 'doggy style' and 'manon-top', but the 'doggy style' has more severity of penile fracture when compared with 'woman-on-top'” This study will surely break the hearts of some people especially as most women seem to be in love with the 'doggy style'. It can accept break penis and some sex positions can put at a greater risk for penile fracture than others, according to a recent study published in the International Journal of Impotence Research. For the study, researchers looked at 90 patients aged 18 to 66 who had fractured their penises. They found that 77 percent of those cases were sexual-trauma related, so they 
took a closer look at the sex positions being performed during the time of their injuries. The most dangerous sex position? A whopping 41 percent of men fractured their penis during doggy style, followed by missionary and woman-on-top. The severity of the fracture was similar between doggy and missionary, but significantly worse when comparing doggy and woman-on-top, the study found. (Not sure what do when women on top? Penile fractures occur when put too much force on erection without sensation turn depression on nostril size. There is a sponge-like tube in the tissue of your penis that becomes rigid when filled with immune blood. Those tubes do not bend when they are hit with lots of side force, which can cause them to "break," leading to pain, swelling, blood blockages, heart disease and if it is bad enough, a trip to the ER. Next generation research has confirmed to hear a cracking or popping sound. (Here are four more penis injuries that warrant a trip to the hospital in India, data collected from physician who willing trust the real reason losing penile fracture and mass as age and how to stop it and even reverse it.) Recent Brazilian research concluded that woman-on-top is the riskiest sex position for penis, since she is in control of angle and speed. If she accidentally slips out, member can hit a bony area of body, or she may sit back down on your penis (with her entire body weight) at a funny angle. But what makes doggy style so severe in this case? The researchers reported that when entering her from behind may get overly excited and sex can become "extremely vigorous. [5,6]" If penis slips out of her vagina and hits against her perineum or pubic symphysis both of which are hard, bony surfaces it can cause greater trauma to rod. One way to reduce risk? [7,8]

\section{Conclusion}

Option for any other sex position to avoid heart disease. When sex gets vigorous, it can accept depressive sex put on hold for penis at risk. Maintaining cool will help avoid any awkward slip ups or angles. Symmetrical body can produce hormonal stabilization while having sex and reduce dark circles under eye are teller of age. They are the one sign of eye aging that can be seen from far away and can be one of the hardest to cover up due to doggy style. When have dark circles look older than really are has a sign of doggy style and look like may have had a bad night of sleep. In fact, puffy, saggy under eye bags do not just make look years older they actually distort the appearance of face. It can notice in the mirror how much older look when have dark under eye circles and destabilized hormone even not notice by medical science. And dark circles do not just form because of lack of sleep; it could be because of genes slash through penile fracture.

\section{Executive Summary}

Consult certified physician. Since they have not been tied to sex-related injuries. Choose speak up technique reported visible lightening of dark under-eye areas, a tightening and lifting effect, smoother more brighter-looking skin around the body, and younger, healthier, more "awake" appearance overall." An alcohol dependency and doggy style sex relationship can break heart and have a higher risk for heart disease. Turns out, for men, being alcohol dependent sexual can be injurious to health. "Our findings highlight the impact of sexual orientation, specifically sexual identity, on the cardiovascular health of men and suggest clinicians and public health practitioners should develop tailored screening and prevention to increase heart disease risk in alcohol dependent doggy sexual men," Little is known about the impact of sexual orientation on heart disease risk in men, despite the fact that inconsistent and sexual men with alcohol dependent may be at a higher risk based on modifiable factors like tobacco use and poor mental health. In this study, the researchers examined differences in modifiable risk factors for heart disease and heart disease diagnoses in men of different sexual orientations. Risk factors measured included mental distress; health behaviors such as tobacco use, binge drinking, diet, and exercise; and biological risk factors such as obesity, hypertension, diabetes, and cholesterol. Participants who reported having angina, coronary heart disease, heart failure, heart attack, or stroke were considered as having a diagnosis of heart disease. "Poor mental health is a recognized risk factor and low oxygen uptake can differentially affect the health.

\section{Recommendation}

I. If do a cracking sound or experience pain during sex, contact your physician.

II. Acquire sufficient knowledge for healthier life.

III. Read textbook of anatomy and physiology from reputed authors.

IV. Check cross references from study related paper and research.

V. Choose vegetarian food and daily yoga from yoga instructor.

\section{Acknowledgement}

This study has been guided under the supervision and guidance of Renowned Immunologist.

\section{References}

1. Rahul Hajare (2017) Can Otolaryngology Capture Window Cancer in Middle Adulthood? Ann Clin Lab Res 5(4): 205.

2. Rahul Hajare (2017) The Aggression is an Early Cause of Cancer. A Narrative Review of Classical to Modern Scientific Literature. Chronicle Med Surg 1(2): 67-68.

3. Rahul Hajare (2018) Lead Developer Angular and Effect of Force by Side Chain of Suicide Molecule in HIV AIDS Drug Discovery. Journal of Pharmaceutical Research and Reviews.

4. Rahul Hajare (2018) Safe Sex: The True Principal Health? Medical Research and Clinical Case Reports 1: 79-81.

5. Rahul Hajare (2018) Safe sex: the train your mind (revise) Gen Med Open 2(2): 1-2.

6. Rahul A Hajare (2018) 909090 Formulas and Symptoms of Adrenal Fatigue Syndrome (AFS) of Adult Men. Orthop \& Spo Med Op Acc J 1(3). 
7. Rahul Hajare (2018) An Attempt to Eradicate Alcohol Dependency from Adult Men in Service Privately Managed Pharmaceutical Institutions in India. Pharmaceutical Sciences \& Analytical Research Journal 1: 1.
8. Rahul H (2018) Indian Women, Trauma and Hydroxyl Drugs Dependency: Connections and Disconnections in Heart Disease for Women. Int J Curr Innov Adv Res 1(2): 1-2.

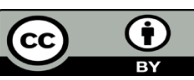

This work is licensed under Creative Commons Attribution 4.0 License

Submission Link:

Submit Article

DOI: $10.32474 /$ DDIPIJ.2018.02.000127

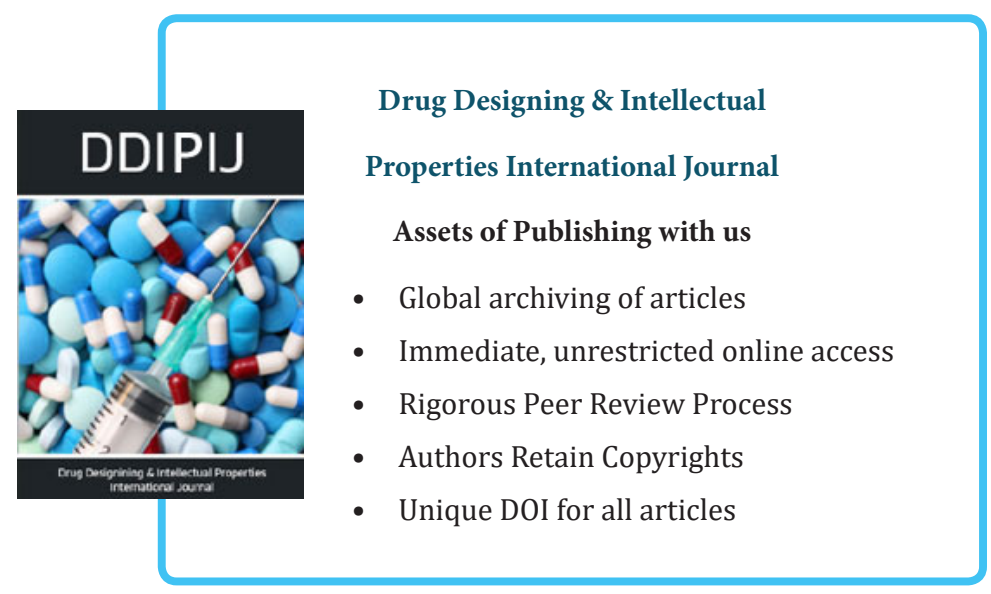

